\title{
IOT and Data Research In Industrial Power Management
}

\author{
B. Pavitra, D.Narendhar Singh
}

\begin{abstract}
IOT plays an important role in collecting data and machine learning for prediction in variety of applications like homecare, healthcare and energy management. In energy management there are various variables such as future power demands, generation status weather conditions and current battery status hard to expect high efficiency.

Here, in this proposed idea, for higher efficiency of renewable energy, an IOT system is needed to monitor and collect these Statuses and provide energy management services. Energy will be consumed of passive operation according to hourly variation in price and battery status will be predicted by using machine learning algorithms like Logistic regression, SVM, and k-NN. We trained the system by considering five random variables in datasheet such as Current time, Current cost, predicted time, predicted cost and Solar battery status from the device. This integrated system is used for uploading power related details of Grid and Solar to IBM cloud. Depending on previous datasheet, analytics will be done by resulting which source has to be triggered to drive the load either Solar or Grid. APIs and NodeRed Tool were used for wiring sensor data and Model predicted output. In future power demands, this design will help to predict the price according to hourly variation based on the units and to trigger the source.
\end{abstract}

Index Terms: $M L$, Logistic regression, SVM, $k-N N$, Node Red Tool, Esp8266.

\section{INTRODUCTION}

Machine learning calculations assume on noteworthy job in software engineering research. The benefit of utilizing these calculations over manual location is that they can bolster human specialists to analyze wellbeing condition, perform examination of complex information in a more productive way. In medicinal services space, information are regularly unpredictable, setting subordinate, and the idea of information is heterogeneous which makes it trying errand for extricating sagacious data from the first information. Machine learning calculations can be joined to distinguish designs in information and the upside of such calculations is that framework can gain from the recognized information which gives more hearty and effective finding. [5]

A large number of machine learning algorithms are available that can perform pattern recognition, classification and regression. Most algorithms are support vector machine (SVM), logistic regression, decision tree, random forest, $\mathrm{k}$ $\mathrm{NN}$ etc. These algorithms are used in different domains applications and each domain uses different performances matrices. In this paper logistic regression, support vector machine (SVM) and k-NN algorithms are applied to know whether the output source is grid or solar. [5]

Revised Manuscript Received on August 14, 2019.

B. Pavitra, Asst.Prof, ECE Department,Anurag Group of Institutions, Hyderabad, T.S, India

D.Narendhar Singh, Asst.Prof, ECE Department,Anurag Group of Institutions, Hyderabad, T.S, India

\section{SYSTEM ARCHITECTURE}

In this project an intelligent energy management system is used to use the renewable energy in an effective manner. The developed integrated system Solar panel is used to charge the battery and based on the variables like current price, current time, predicted price, predicted time, current cost, predicted cost, solar battery status is taken into consideration based on this variables, the output source is triggered.

The variables date and solar battery status are uploaded to cloud and placed in database. The data is given for analysis for with predefined data related to cots, time and battery status using DSX machine service of IBM Cloud. After analysis the values are taken from the cloud and sent to the controller based on the result the loads trigger [1]

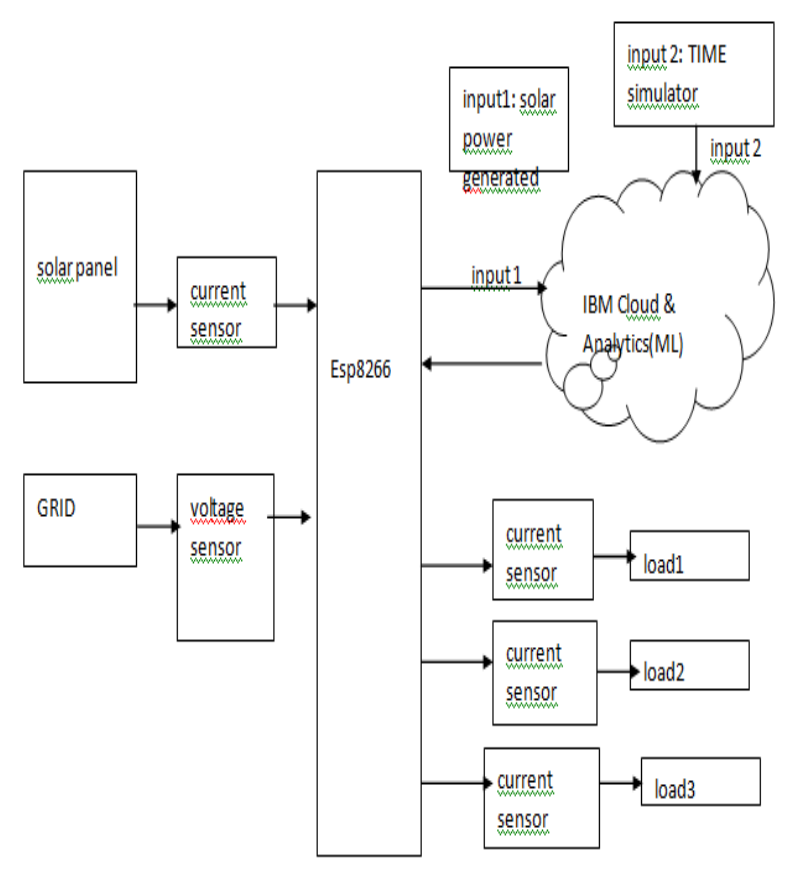

Fig1: Block diagram

\section{HARDWARE DESCRIPTION}

Node MCU (ESP8266):

The ESP8266 fig (2) is used as embedded controller to interface with Solar panel, GRID and connect with to IBM Cloud.

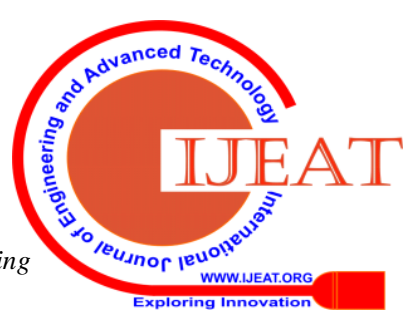


The ESP8266 has 30 pins inbuilt Wi-Fi and BLE module RAM of $512 \mathrm{~kb}$, flash of $16 \mathrm{mb}$, GPIO pins of 36,18 ADC pins, 2 DAC pins, 5 busses (SPI, I2C,UART,I2S, CAN). BP and Temperature sensors are connected to the ESP32 and sensors output values are sent cloud.

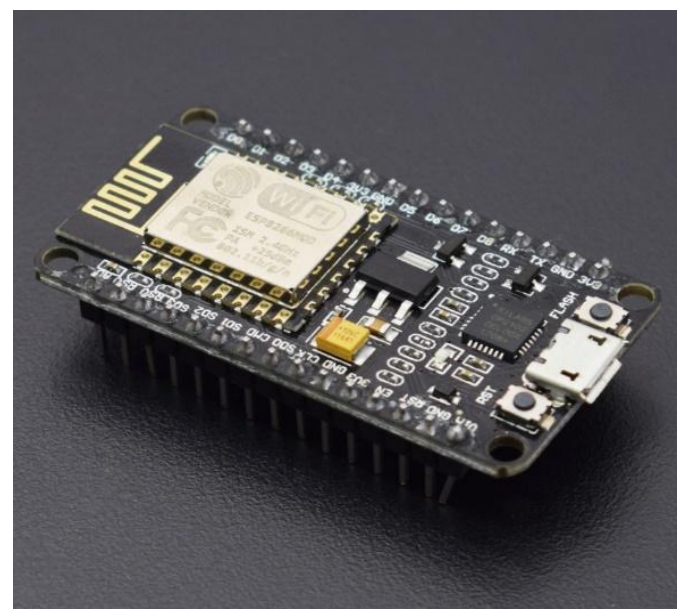

Fig 2: ESP8266 controller

\section{SOLAR PANEL:}

Solar panel is one which converts sunlight into electric power; solar cells in the solar panel produce direct current from light that can be used to the power equipment or to recharge the battery.

Here the cable type is PV insulated, sheath and UV resistance cable and material is made of copper the panel is 20W solar panel (Pmax)-20W+-3\% and open circuit voltage(Voc)-21.5V and short circuit current(Isc)-1.30 A voltage at maximum power( $\mathrm{Vmp})$ is $17.7 \mathrm{~V}$ and current at maximum power(Imp) is $1.13 \mathrm{~A}$ and maximum system voltage is $600 \mathrm{~V}$

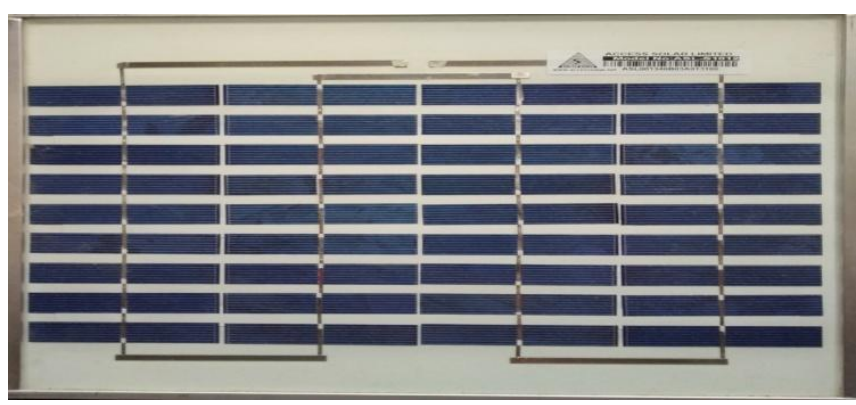

Fig 3: Solar panel

\section{GRID (Set-Up transformer):}

The step-up transformer is used in this project and for step-up transformer output is greater than the input voltage. It can easily multiply and divide the voltage \& Current in AC circuits.

The Step-up transformer can make a long distance transmission of electric power and the AC voltage can be stepped up and the current is stepped down for reduced wire resistance power losses along power lines connecting generating stations with loads.

Here the working voltage is $12 \mathrm{~V} / 1 \mathrm{Amp}$ and the transformer has $240 \mathrm{~V}$ primary windings and centre tapped with 32-bit processer, two cores, CPU frequency of $160 \mathrm{mhz}$,

secondary windings. This is a general purpose chassis mounting mains transformer.

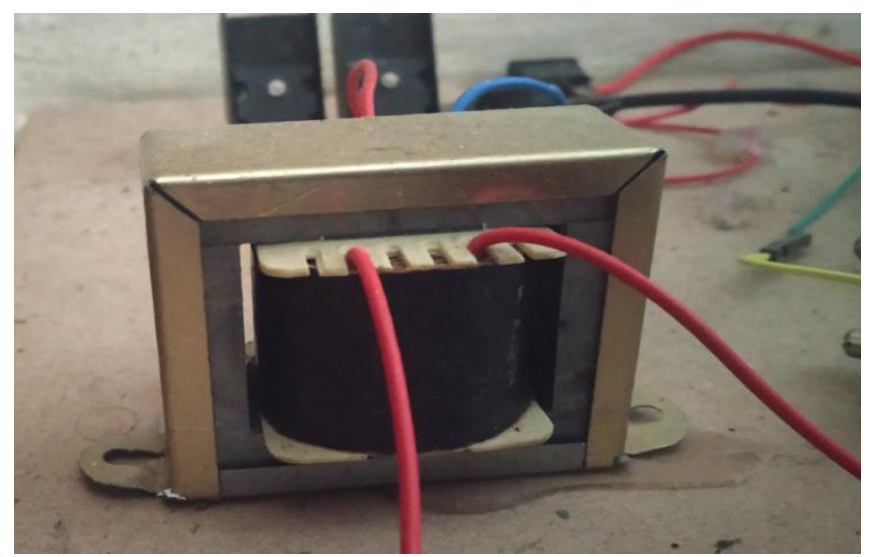

Fig 4: GRID (Set-Up transformer)

\section{SOFTWARE:}

ARDUINO IDE: The Arduino IDE is an open source software where we can write, execute and upload to the board it can install for windows, Linux, etc., here different languages like $\mathrm{C}, \mathrm{C}++$, embedded $\mathrm{C}$ are used. I have written the program in embedded $\mathrm{C}$ and uploaded to hardware board by connecting USB. The functions of IDE are setup () are used to execute or reset the program, loop () are used to repeat a specific block of code in the program.

PYTHON: Python is an interpreted, object -oriented, high -level programming language with dynamic semantics. It is high level built in data structures, combined with dynamic typing and dynamic binding make it very attractive for Application development.

Python is simple, easy to learn syntax. Debugging python programs is easy; the edit-test-debug cycle is fast.

IBM CLOUD: Cloud storage enables applications to upload data to network of remote, connected servers. Applications can maintain that data and access it from any ware, applications access data using a web based API that works with client applications.

\section{V.SYSTEM IMPLEMENTATION:}

The experimental works of this project conducted on three different Machine Learning Algorithms i.e. logistic regression, Support vector machine (SVM), k-NN to find out the best method for predicting output source.

Independent parameters such as Current time, Current cost, predicted time, predicted cost and Solar battery status from the device are observed and based on the parameters the Analysis is done and the output source is triggered. There are up to 250 data inputs taken and observed. From those values we create a csv file, that file is uploaded to IBM cloud via Wi-Fi for analysis. For each parameters data, each of the of this independent data is randomly divided into two datasheets i.e. Training and Testing datasheets.

The Training dataset consists of 190 and testing dataset consists of 60 cases. 


\begin{tabular}{|c|c|c|c|c|c|c|c|c|c|c|}
\hline S.No & $\mathbf{C T}$ & $\mathbf{C P}$ & PT & PP & U & CC & PC & DC & SB & ST \\
\hline 1 & 6 & 5 & 8 & 9 & 10 & 50 & 90 & -40 & 100 & $\mathrm{~S}$ \\
\hline 2 & 6 & 5 & 8 & 9 & 10 & 50 & 90 & -40 & 90 & $\mathrm{~S}$ \\
\hline 3 & 6 & 5 & 8 & 9 & 10 & 50 & 90 & -40 & 80 & $\mathrm{~S}$ \\
\hline 4 & 6 & 5 & 8 & 9 & 10 & 50 & 90 & -40 & 70 & $\mathrm{~S}$ \\
\hline 5 & 6 & 5 & 8 & 9 & 10 & 50 & 90 & -40 & 60 & $\mathrm{~S}$ \\
\hline 6 & 6 & 5 & 8 & 9 & 10 & 50 & 90 & -40 & 50 & $\mathrm{~S}$ \\
\hline 7 & 6 & 5 & 8 & 9 & 10 & 50 & 90 & -40 & 40 & $\mathrm{G}$ \\
\hline 8 & 6 & 5 & 8 & 9 & 10 & 50 & 90 & -40 & 30 & $\mathrm{G}$ \\
\hline 9 & 6 & 5 & 8 & 9 & 10 & 50 & 90 & -40 & 20 & $\mathrm{G}$ \\
\hline 10 & 6 & 5 & 8 & 9 & 10 & 50 & 90 & -40 & 10 & $\mathrm{G}$ \\
\hline 11 & 6 & 9 & 8 & 5 & 10 & 90 & 50 & 40 & 100 & $\mathrm{~S}$ \\
\hline 12 & 6 & 9 & 8 & 5 & 10 & 90 & 50 & 40 & 90 & $\mathrm{~S}$ \\
\hline 13 & 6 & 9 & 8 & 5 & 10 & 90 & 50 & 40 & 80 & $\mathrm{~S}$ \\
\hline 14 & 6 & 9 & 8 & 5 & 10 & 90 & 50 & 40 & 70 & $\mathrm{~S}$ \\
\hline 15 & 6 & 9 & 8 & 5 & 10 & 90 & 50 & 40 & 60 & $\mathrm{~S}$ \\
\hline 16 & 6 & 9 & 8 & 5 & 10 & 90 & 50 & 40 & 50 & $\mathrm{~S}$ \\
\hline 17 & 6 & 9 & 8 & 5 & 10 & 90 & 50 & 40 & 40 & $\mathrm{G}$ \\
\hline 18 & 6 & 9 & 8 & 5 & 10 & 90 & 50 & 40 & 30 & $\mathrm{G}$ \\
\hline 19 & 6 & 9 & 8 & 5 & 10 & 90 & 50 & 40 & 20 & $\mathrm{G}$ \\
\hline
\end{tabular}

Table 1: Data set (.csv file)

CT-Current Time, CP-Current Price, PT-Predicted Time, PP-Predicted Price, U-Units, CC-Current Cost, PCPredicted Cost, DC-Difference Cost, SB-Solar battery, STStatus,S-Source,G-Grid.

With the above data sets, we are creating a ML model that can predict the output source. The Classification Accuracy rate of Logistic regression, SVM, k-NN algorithms using sensors variables are given below table 2

Depending on the accuracy percentage we can finalize which ML algorithm is predicting the best output source.

We will write the code in python language in jupyter note book, and create the machine learning model token. Then we go to NODE-RED service in that service we create a flow chart for sensors data and ML model predicted output. The below fig (5) shows the flowchart for output by using Node Red .

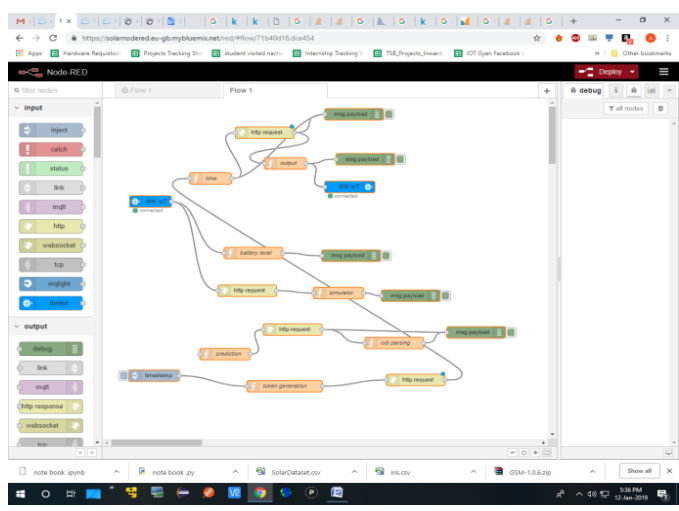

Fig (5): Node-RED output

\section{VI. .RESULTS}

The accuracy for different ML algorithms is executed using the python code in jupyter notebook with table 1 .csv.file data set. From that I have taken SVM algorithm as better predicted algorithm for this project.

From Table 1 ST-Status when the output is grid power then both the loads are triggered shown in the below fig 6

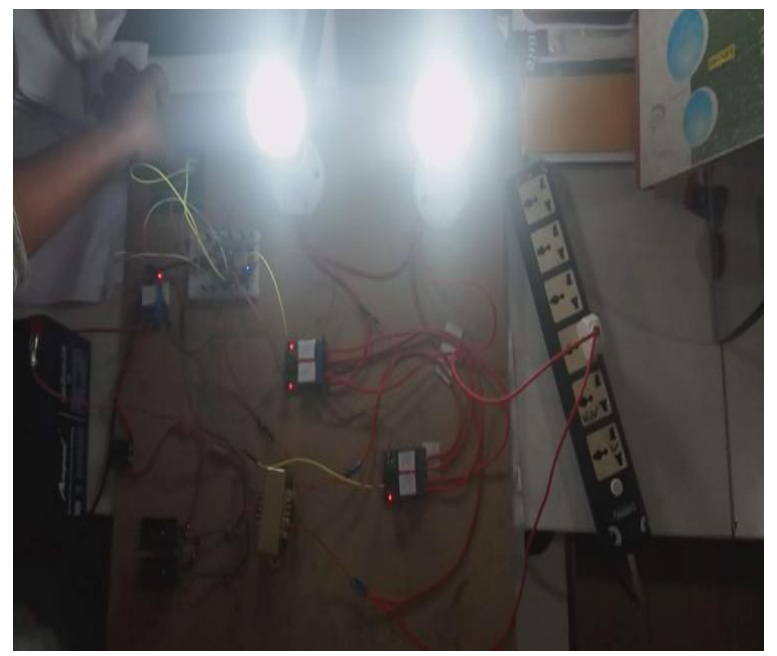

Fig 6: Grid power is used two loads triggering

After some time the values are monitired again and based on the vaues the solar power is used and the priority load is trggred shown as fig 7

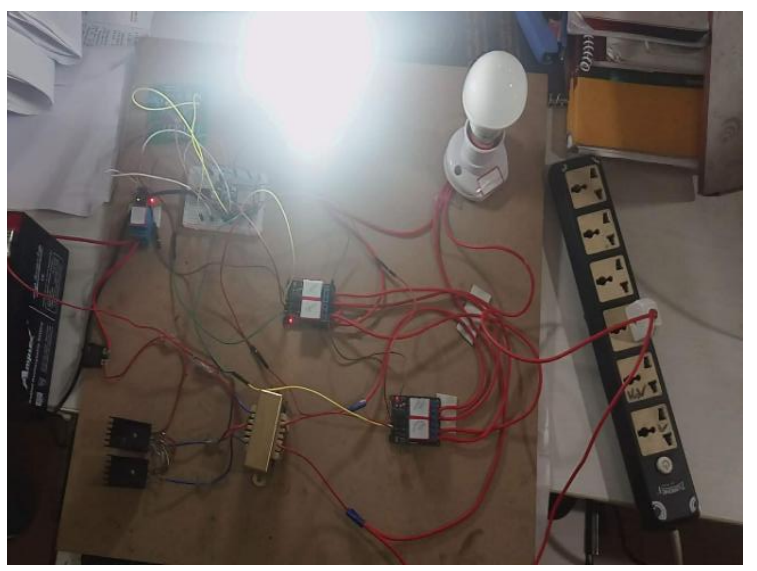

Fig 7: Solar power is used priority load is triggered.

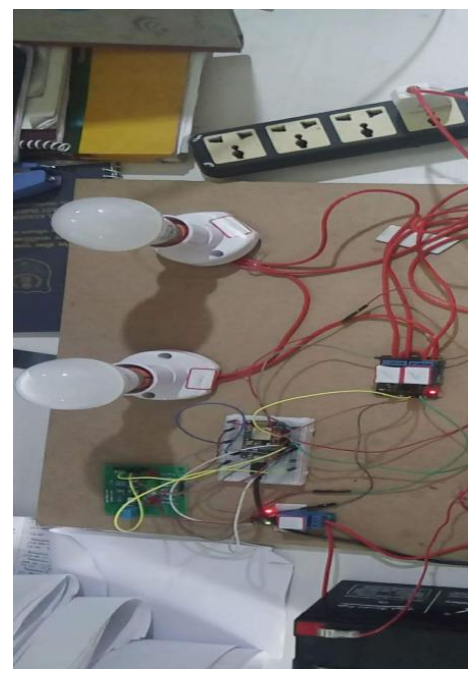

Fig 8:Project prototype

Published By: 


\section{CONCLUSION AND SCOPE}

In future there will be large demand for power, keeping this in mind I have designed an efficient power management system in which the renewable energy is used so that the power consumption and also the cost can be reduced.

This paper provides a very efficient usage of Renewable energy in a way that the cost is reduced and also the power is consumed in effective manner which results for the better future and green power management.

When solar power is not available or not sufficient, grid is triggered to drive load and maximum during peak hours, the solar power is available, so the grid

is not triggered but solar is triggered. The selection of source and load is decided by model developed using DSX service of IBM cloud.

The variations in the costs and power metrics are the major challenges which can overcome by continuous monitoring and colleting large number of data for these variables so that in future the accuracy can be increased more which gives the better results.

\section{REFERENCES:}

1. Internet of Things for Smart Cities 2014. Zanella, A., Bui, N., Castellani, A., Vangelista, L., \& Zorzi, M.

2. An Internet of Things Framework for Smart Energy Buildings: Designs, Prototype, and Experiments.2015 IEEE. Pan, J., Jain, R., Paul, S., Vu, T., Saifullah, A., \& Sha, $M$.

3. A Parallel and Incremental Approach for Data-Intensive Learning of Bayesian Networks 2015. Yue, K., Fang, Q., Wang, X., Li, J., \& Liu.

4. Parameter-Based Data Aggregation for Statistical Information Extraction in Wireless Sensor Networks.2010. IEEE Transactions on vehicular technology Engineering. Jiang, H., Jin, S., \& Wang, C.

5. Enabling Smart Grid with ETSI M2M Standards. 2012. Lu , G., Seed, D., Starsinic, M., Wang, C., \& Russell, P. (n.d.). 